\title{
USING MOBILE LASER SCANNING POINT CLOUDS TO EXTRACT URBAN ROADSIDE TREES FOR ECOLOGICAL BENEFITS ESTIMATION
}

\author{
Wen Fan ${ }^{1}$, Bisheng Yang ${ }^{1, *}$, Fuxun Liang $^{1}$, Zhen Dong $^{1}$ \\ ${ }^{1}$ State Key Laboratory of Information Engineering in Surveying, Mapping and Remote Sensing, Wuhan University, Wuhan 430079, \\ China (rswfan, bshyang, liangfuxun, dongzhenwhu) @whu.edu.cn
}

Commission II, WG II/3

KEY WORDS: Point clouds, Classification, Min-cut, Tree segmentation, Living Vegetation Volume (LVV)

\begin{abstract}
:
Roadside trees in the city play a crucial role in addressing the issues of air pollution, urban heat island effects, road noise, and so on. This paper proposes an efficient and robust method to automatically extract individual roadside trees with morphological parameters from mobile laser scanning (MLS) point clouds for ecological benefits estimation. The proposed method consists of four steps: MLS data pre-processing, pole-like objects classification, individual tree extraction, morphological parameters calculation for ecological benefits estimation. The proposed method is verified using three complex datasets in Shanghai, China. Comprehensive experiments demonstrate that the proposed method achieves good performance in extracting individual tree in terms of average precision and recall $(91.83 \%, 92.60 \%)$, and provides detailed information for ecological benefits estimation.
\end{abstract}

\section{INTRODUCTION}

Roadside trees are essential in urban environment. Location and parameter measurements of urban vegetation are important for improving urban ecological environment and maintaining human mental health (Wang et al., 2020). At the same time, roadside trees can improve the air quality through carbon sequestration and have a direct impact on the degree of urban afforest (Zhao et al., 2018). The researchers find that the degree of greening can change the mental health of residents. Many scholars have extracted trees based on airborne laser scanning (ALS) system and terrestrial laser scanning (TLS) system in the past years (Baptista et al., 2018, Williams et al., 2019). Researchers focus on urban green space information, forest carbon storage and biomass. The tops of roadside trees can be well observed by ALS system. However, the lack of observation at the bases of the trees limits the application of roadside trees to street scene. Terrestrial laser scanner has high sampling density and rich information, but poor with mobility, so it is difficult to obtain the scene information on both sides of the road quickly. Mobile laser scanning (MLS) system has the advantages of high efficiency and high density, MLS plays a very important role in high-definition map, automatic driving, highway reconstruction and expansion, urban 3D modeling and other engineering applications. With high precision, MLS can directly obtain the three-dimensional spatial information of the street. Thus, MLS provides rich data for trees morphological parameters estimation, which provides support for the city digitization.

Some scholars extract and quantify the information of roadside tree from MLS data. (Lehtomäki et al., 2010) detects pole-like objects from MLS data. By carrying out scanning line segmentation, re-clustering, and clustering combination, pole-like objects categories such as light pole, traffic sign and tree trunk can be obtained through classification. However, the slanted objects are lost in the detection, and the accuracy is affected by point clouds of buildings, which leads to misclassification.

\footnotetext{
* Corresponding author
}

(Rutzinger et al., 2011) uses 3D Hough and region growth to divide point clouds into multiple sections, and combines sections to extract street trees. However, the time complexity is high. (Li et al., 2016) obtains tree clusters by a dual growing method and individual tree by segmenting overlapping crowns based on the consistency with area increment. (Guan et al., 2016) proposes a tree segmentation method based on Euclidean distance clustering and Voxel-based Normalized Cut (VNCut) segmentation approach. But the extraction of trees in complex scenes is not well treated. (Liu et al., 2017) extracts the crown seeds in the initial layer by comparing the number of midpoints in each layer, then carries out horizontal growth in the crown area to obtain the tree extraction results, but the case of overlapping crowns is not considered. Moreover, this method is lack of further research and application till now. (Chen et al., 2019) gets an individual tree by splitting it first and then classifying it, with tests of four different segmentation methods for extraction of single tree clusters (Euclidean distance clustering, region growing segmentation, normalized cut and supervoxelbased segmentation) and two classification methods (k-NN and Random forests)

The method of this paper is divided into four steps, which includes (1) MLS point clouds pre-processing, (2) Pole-like objects extraction, (3) Individual tree extraction and (4) morphological parameters calculation and ecological benefits estimation. The rest of this article is arranged as follows. Section 2 introduces the MLS data tested in the experiment. Section 3 describes the methods of tree location and extraction. Section 4 is the experimental results and discussion. Finally, Section 5 summarizes this paper. The main contributions of the proposed method are as follows:

(1) The confidence of each pole belonging to trunk is calculated and considered as the order of hierarchical min-cut, which can make sure that poles more likely to be trees can be cut first, so that the extracted results are more complete. This approach can effectively avoid unexpected removal of target poles. 
(2) The Living Vegetation Volume and annual $\mathrm{O}_{2}$ output, $\mathrm{CO}_{2}$ and $\mathrm{SO}_{2}$ absorption, dust retention, transpiration amount are calculated at individual tree level and block level, providing detailed information to estimate ecological benefits of urban roadside trees.

\section{MOBILE LASER SCANNING TEST DATA}

The test data is collected by Alpha3D mobile system. The mobile laser scanning measurement system integrates RIEGL VUX-1 laser scanner with high precision and long measuring range, global navigation satellite system (GNSS) receiver and high precision inertial measurement unit (IMU). In the range of $100 \mathrm{~m}$ from scanner to point, the relative accuracy of the collected data is about $8 \mathrm{~mm}$.

The research areas are located in three areas of Shanghai, namely Huangpu District, Fengxian District and Zhangjiang high-tech park. Huangpu District have long history with a wide variety of data objects and complex scenes, including buildings, telephone poles, road trees, pedestrians, vehicles and railings, leading to data occlusion. Zhangjiang District is a new district with simple scene and good data quality, but there are many overlapping tree canopies. Fengxian District is in between. Table 1 shows a detailed description of these three datasets.

\section{METHODOLOGY}

In order to eliminate the influence of unrelated objects, this paper adopts the method of first segmentation and then classification to extract the complete roadside trees. The pole with obvious geometric property is used as the foreground point and the complete object is segmented by min-cut. At last, the object point cloud of an individual tree is preserved as much as possible. The pipeline of the proposed method is illustrated in Figure.1. The key steps of the proposed method are described in detail as follows.

\subsection{MLS point clouds pre-processing}

The data collected by the measurement vehicle is the complete point clouds obtained under its driving track, which includes a large number of redundant data, such as ground, buildings, etc., which account for more than $65 \%$ of the total scene point clouds. The raw data needs to be pre-processed to speed up the segmentation speed of the target objects in the experiment. It includes original data segmentation, point clouds subsampling, ground points removal, large-scale man-made objects removal and refinement.

In this paper, a fast ground filtering method is used. This method is implemented as follows:

1) The point clouds are projected on a two-dimensional $X Y$ plane, and the points on the plane are gridded according to a certain resolution $R_{0}$;

2) The minimum number threshold of grid points is setting as $N_{\text {thresh }}$. If the number of points in the grid is less than $N_{\text {thresh }}$, it is considered that the interior is noise point and needs to be filtered;

3) The height difference between the lowest point and the candidate point in the grid is calculated, $H_{\text {diff }}$. If $H_{\text {diff }}$ is less than the threshold $H_{\text {thresh }}$, the point will be filtered out, and all the filtered points in the voxel will be accumulated to get the ground points.

In the point cloud of street scene, buildings, railings and other surface distribution features also cause a lot of redundancy. In this paper, large patches are removed by the following methods:

1) The kd-tree structure is established to store all the points, which is used to search the nearest point in $R_{\text {cur }}$ neighborhood of seed points. Calculating the curvature of each point and sorts it.

2) The minimum curvature point is taken as the initial seed point $P_{i}$ (i $=0,1, \ldots, \mathrm{M}$, where $\mathrm{M}$ is the number of unsegmented points). The cosine value of the angle between the plane equation of the seed point is calculated, the current neighborhood point and the normal vector of the initial seed point, and the vertical distance from the neighborhood point to the plane of the current seed point. The points that meet the angle threshold and distance threshold are included in the current plane of the seed point, and continue to grow as the face points, and the points are marked as split points. After all neighborhood points of the current seed point are searched, delete the seed point.

3) After all seed points have finished growing, the plane with less than $M_{\text {plane }}$ points is considered too small to be deleted. In this paper, only large-scale man-made patches are deleted in this process.

4) The removed patches are refined. A buffer of a certain width is established for the obtained patches to remove the points in the buffer. The refining process is necessary because there are doors and windows on one side of the building facade, which will produce many miscellaneous points in the process of scanning.

\subsection{Pole-like objects extraction}

Firstly, we extract the pole-like point clouds by slicing the height of the pre-processed object point clouds. A slice with a certain thickness can be obtained. The Euclidean distance clustering is applied to the slices. The discontinuous clusters are removed by connectivity analysis. When the number of connected clusters is larger than the preset threshold, the roundness is estimated, and the formula is shown in eqs 1 . Only the cluster whose $C_{i}$ ( $\mathrm{i}=0,1, \ldots, \mathrm{M}$, where $\mathrm{M}$ is the number of clusters) is larger than $C_{\text {thresh }}$ is preserved as the initial cluster.

$$
C_{i}=\frac{\text { Area }_{i}}{\pi *\left(D_{i} / 2\right)^{2}}
$$

Where $C_{i}$ is the quasi-circularity of the cluster, and $C_{\text {thresh }}$ is the threshold of quasi-circularity. $D_{i}$ is the diagonal length of the main direction bounding box of the subset projected on the horizontal plane, and $\mathrm{Area}_{i}$ is the convex hull area of the subset projected on the horizontal plane XY. In order to extract the remaining structure of pole-like point clouds, the random forest soft classification method is used to estimate the current pole-like point clouds. The confidence of each pole belonging to trunk is calculated and considered as the order of hierarchical min-cut, which can make sure that poles more likely to be trunks can be cut first, so that the extracted results are more complete. Soft classification features are selected according to 


\begin{tabular}{|c|c|c|c|}
\hline Dataset & Data size & Road Length & Coverage Area \\
\hline I. Huangpu Dataset & $600 \mathrm{M}$ & $495 \mathrm{~m}$ & $27225 \mathrm{~m}^{2}$ \\
II. Zhangjiang Datase & $600 \mathrm{M}$ & $443 \mathrm{~m}$ & $63792 \mathrm{~m}^{2}$ \\
III. fengxian Dataset & $648 \mathrm{M}$ & $648 \mathrm{~m}$ & $94608 \mathrm{~m}^{2}$ \\
\hline
\end{tabular}

Table 1. Datasets description

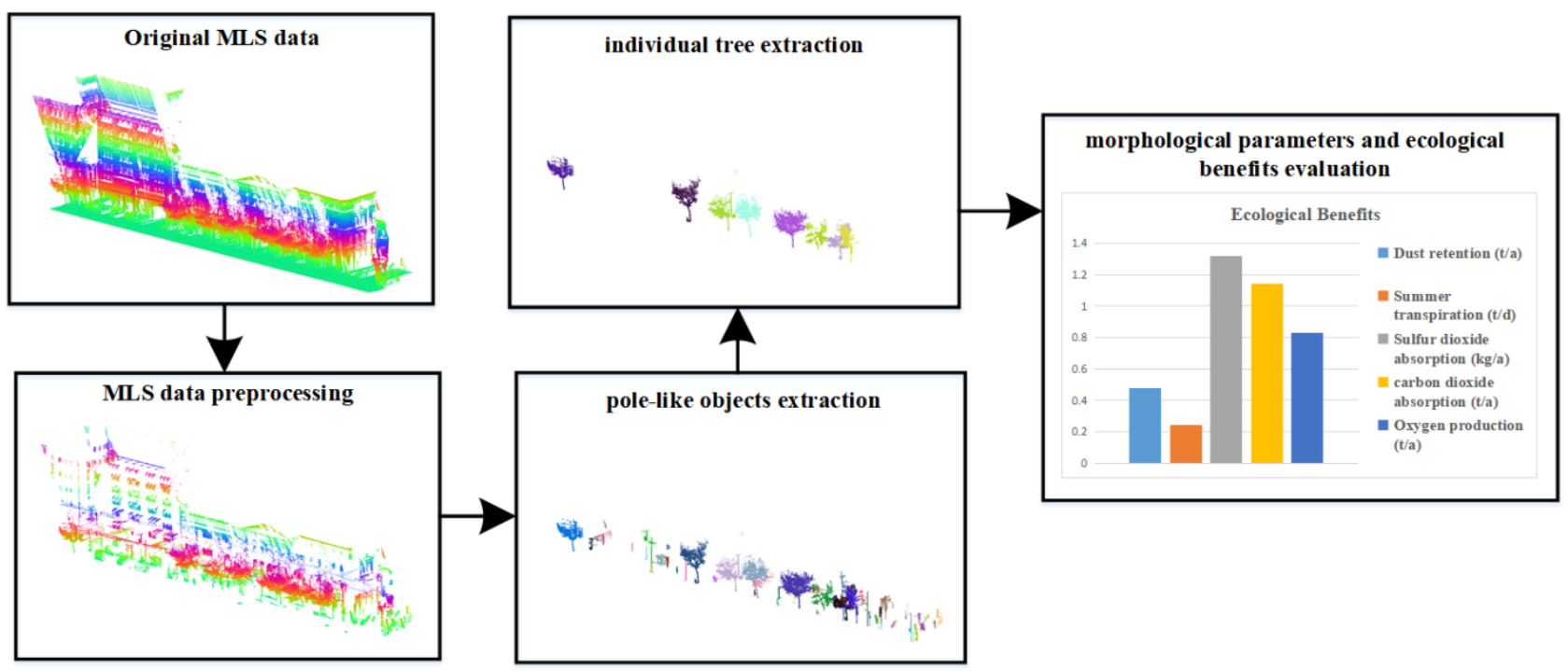

Figure 1. The workflow of the proposed method.

Table 2. During the experiment, since only the geometric properties of the poles are similar, the top neighborhood points of the poles are added for soft classification. Then by applying mincut method, segmentation operation is carried out using pole point clouds we obtained as prior information. By referring to the method of (Yang et al., 2016), the residual structure of polelike objects is extracted by min-cut, and a complete pole-like object is obtained. In the process of min-cut, the current pole is set as the foreground point, and the nearest pole is set as the background point.

\subsection{Individual tree extraction}

Local and overall features are calculated based on the morphological characteristics. Trees are extracted from the pole-like objects using a random forest. The design of local features refers to the work of (Weinmann et al., 2015). This local feature set encapsulates linearity, planarity, scattering, omnivariance, anisotropy, eigenentropy and change of curvature. For all 1760 training samples in the experiment, the accuracy of cross validation is $95.6 \%$.

\begin{tabular}{|c|c|}
\hline Local feature & formula \\
\hline linearity & $L_{\lambda}=\frac{\lambda_{1}-\lambda_{2}}{\lambda_{1}}$ \\
planarity & $P_{\lambda}=\frac{\lambda_{2}-\lambda_{3}}{\lambda_{1}}$ \\
scattering & $S_{\lambda}=\frac{\lambda_{3}}{\lambda_{1}}$ \\
omnivariance & $O_{\lambda}=\sqrt[3]{\lambda_{1} * \lambda_{2} * \lambda_{3}}$ \\
anisotropy & $A_{\lambda}=\frac{\lambda_{1}-\lambda_{3}}{\lambda_{1}}$ \\
eigenentropy & $E_{\lambda}=-L_{\lambda} \ln L_{\lambda}-P_{\lambda} \ln P_{\lambda}-S_{\lambda} \ln S_{\lambda}$ \\
change of curvature & $C_{\lambda}=\frac{\lambda_{3}}{\lambda_{1}+\lambda_{2}+\lambda_{3}}$ \\
\hline
\end{tabular}

Table 2. The calculation of local features.

According to the point set $X_{i}(\mathrm{i}=0,1, \ldots, \mathrm{M}$, where $\mathrm{M}$ is the number of points), the corresponding covariance matrix $\mathrm{S}$ can be obtained, which is a symmetric positive definite matrix with three non-negative eigenvalues $\lambda_{1}, \lambda_{2}, \lambda_{3}\left(\lambda_{1}>\lambda_{2}>\lambda_{3} \geq 0\right)$.

$$
S=\frac{1}{k+1} \sum_{i=0}^{M}\left(X_{i}-\bar{X}\right)\left(X_{i}-\bar{X}\right)^{T}
$$

The three eigenvalues of the covariance matrix represent the orthogonal eigenvectors in the three principal directions of the point set ellipsoid. Linearity, planarity and scattering can represent the dispersion degree of the set of points in different directions. The dispersion, information and flatness of natural structure are obviously different from that of artificial structure. It can be expressed by omnivariance, anisotropy, eigenentropy and change of curvature.

The overall features include the height of the object, the diameter of the object, the minimum bounding box diameter of the pole, and the diameter of the auxiliary structure. The feature combination is tested with SVM and compared with the calibrated truth value. Through feature selection and experiments, the local and global features selected in this paper can effectively describe the characteristics of objects.

\subsection{Morphological parameters calculation and ecological benefits estimation}

Four morphological parameters are calculated for each tree: tree height $(\mathrm{H})$, crown width (D), crown height (h) and diameter at breast height $(\mathrm{DBH}) . \mathrm{DBH}$ is calculated by fitting the diameter of the circle at the trunk $1.2-1.4 \mathrm{~m}$ above the ground. The ecological benefits of trees are estimated by living vegetation volume (LVV). LVV is calculated by $h$ and D. It breaks through the limitation of the original two-dimensional green index. The calculation method of LVV is shown in eqs 3 . According to the 
calculation results of LVV, combined with the standard conversion amount of green environmental benefits based on LVV by the research group of "Shanghai greening 3D Remote Sensing Survey", annual $\mathrm{O}_{2}$ output, $\mathrm{CO}_{2}$ and $\mathrm{SO}_{2}$ absorption, dust retention and transpiration amount are calculated. These parameters can directly reflect the ecological benefit level of the urban scene.

$$
L V V=\frac{\pi * h * D^{2}}{6}
$$

The eqs 3. is applied for the tree crowns with elliptical, spherical or hemispherical shapes.

\section{RESULTS AND DISCUSSION}

The vehicle laser scanning system collects three datasets, covering three different urban areas of Huangpu District, Zhangjiang District and Fengxian District in Shanghai, China respectively. Figure 2(a) shows the data set of a 495m road in Huangpu District, which is a relatively complex urban scene, including buildings, power poles, street trees, pedestrians, vehicles, railings and other objects. The tree canopy is interspersed with wires. Figure 2(c) shows the data set of Fengxian District, and Figure 2(e) shows the data set of Zhangjiang District. The data sets of Zhangjiang District and Fengxian District contain roadside trees, shrubs, traffic signs, street lights and other objects. The high degree of tree overlap brings challenge to individual tree segmentation.

In the experiment, the parameter settings are shown in Table 3 , and the results are evaluated quantitatively. The experiment shows that the proposed method extracts individual trees with the precision and recall in terms of $89.47 \%$ and $96.23 \%, 91.95 \%$ and $91.33 \%, 94.07 \%$ and $90.24 \%$, respectively. The corresponding time cost is 583s, 592s, 540s. Most of the trees are extracted completely and quickly, which proves the universality and practicability of the algorithm. The experimental results from the three districts are listed in Figure 2(b) (d) (f).

\begin{tabular}{|c|c|c|}
\hline Parameters & Description & Values \\
\hline$R_{0}$ & Grid resolution & $0.3 \mathrm{~m}$ \\
$N_{\text {thresh }}$ & Minimum number of grid points & 50 \\
$H_{\text {diff }}$ & Height difference threshold & $0.2 \mathrm{~m}$ \\
$R_{\text {cur }}$ & Kd-tree search radius & $0.3 \mathrm{~m}$ \\
$M_{\text {plane }}$ & Minimum number points of plane & 500 \\
$A_{\text {theresh }}$ & Point to plane angle threshold & $0.174 \mathrm{rad}$ \\
$D_{\text {theresh }}$ & Point to plane distance threshold & $0.4 \mathrm{~m}$ \\
$C_{\text {thresh }}$ & quasi-circularity estimation threshold & 0.5 \\
\hline
\end{tabular}

Table 3. Parameters settings for two experimental datasets

The corresponding LVV is $707.50 \mathrm{~m}^{3}, 2714.13 \mathrm{~m}^{3}$, and $8361.88 \mathrm{~m}^{3}$, the $\mathrm{O}_{2}$ output, $\mathrm{CO}_{2}$ absorption amount and $\mathrm{SO}_{2}$ absorption amount are 22.39t/a, 30.87t/a, 35.70kg/a, respectively. Moreover, the annual dust retention amount is $12.96 \mathrm{t}$, the transpiration amount in summer is $6.48 \mathrm{t} / \mathrm{d}$.

\section{CONCLUSION}

In this paper, a method is proposed to locate and measure the trees from the MLS point clouds, and estimate the ecological benefits of roadside trees. In order to reduce the storage and calculation of redundant points, the original data is pre-processed. Based on the candidate points, the first step is to segment the pole-like objects, and then to classify the objects into two categories to get the tree object. Through the complete roadside tree object, three parameters are measured, and the ecological benefits of trees are estimated by LVV. In the experiment, MLS data of three different scene complexity regions in Shanghai are selected. Through the quantitative evaluation of the experimental results, it is proved that this method is accurate and efficient for the extraction and measurement of the roadside tree in MLS data.

\section{ACKNOWLEDGEMENTS}

This research is jointly supported by the National Natural Science Foundation of China Projects ( No.41531177, No. 41725005)

\section{REFERENCES}

Baptista, M. D., Livesley, S. J., G Parmehr, E., Neave, M., Amati, M., 2018. Terrestrial laser scanning to predict canopy area metrics, water storage capacity, and throughfall redistribution in small trees. Remote Sensing, 10(12), 1958.

Chen, Y., Wang, S., Li, J., Ma, L., Wu, R., Luo, Z., Wang, C., 2019. Rapid Urban Roadside Tree Inventory Using a Mobile Laser Scanning System. IEEE Journal of Selected Topics in Applied Earth Observations and Remote Sensing, 12(9), 36903700.

Guan, H., Cao, S., Yu, Y., Li, J., Liu, N., Chen, P., Li, Y., 2016. Street-Scene Tree Segmentation from Mobile Laser Scanning Data. International Archives of the Photogrammetry, Remote Sensing and Spatial Information Sciences, 3.

Lehtomäki, M., Jaakkola, A., Hyyppä, J., Kukko, A., Kaartinen, H., 2010. Detection of vertical pole-like objects in a road environment using vehicle-based laser scanning data. Remote Sensing, 2(3), 641-664.

Li, L., Li, D., Zhu, H., Li, Y., 2016. A dual growing method for the automatic extraction of individual trees from mobile laser scanning data. ISPRS Journal of Photogrammetry and Remote Sensing, 120, 37-52.

Liu, J., Xiao, Z., Chen, Y., Huang, P., Wu, R., Li, J., 2017. Automated extraction of urban roadside trees from mobile laser scanning point clouds based on a voxel growing method. 2017 IEEE International Geoscience and Remote Sensing Symposium (IGARSS), IEEE, 1832-1835.

Rutzinger, M., Pratihast, A. K., Oude Elberink, S. J., Vosselman, G., 2011. Tree modelling from mobile laser scanning data, sets. The photogrammetric record, 26(135), 361-372.

Wang, J., Zhou, W., Jiao, M., Zheng, Z., Ren, T., Zhang, Q., 2020. Significant effects of ecological context on urban trees' cooling efficiency. ISPRS Journal of Photogrammetry and Remote Sensing, 159, 78-89.

Weinmann, M., Jutzi, B., Hinz, S., Mallet, C., 2015. Semantic point cloud interpretation based on optimal neighborhoods, relevant features and efficient classifiers. ISPRS Journal of Photogrammetry and Remote Sensing, 105, 286-304.

Williams, J., Schönlieb, C.-B., Swinfield, T., Lee, J., Cai, X., Qie, L., Coomes, D. A., 2019. 3D Segmentation of Trees Through a Flexible Multiclass Graph Cut Algorithm. IEEE Transactions on Geoscience and Remote Sensing. 
Yang, B., Dai, W., Dong, Z., Liu, Y., 2016. Automatic forest mapping at individual tree levels from terrestrial laser scanning point clouds with a hierarchical minimum cut method. Remote Sensing, 8(5), 372.

Zhao, Y., Hu, Q., Li, H., Wang, S., Ai, M., 2018. Evaluating carbon sequestration and PM2. 5 removal of urban street trees using mobile laser scanning data. Remote Sensing, 10(11), 1759.

Revised April 2020 


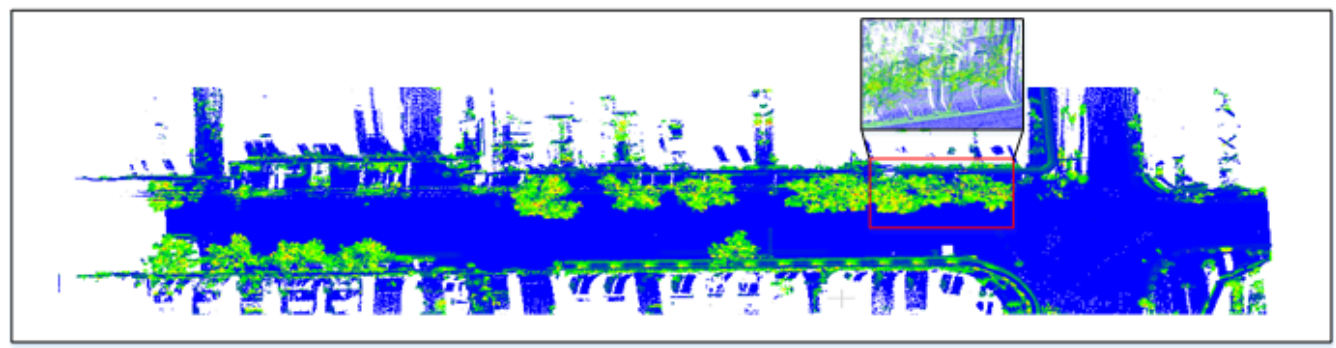

(a)

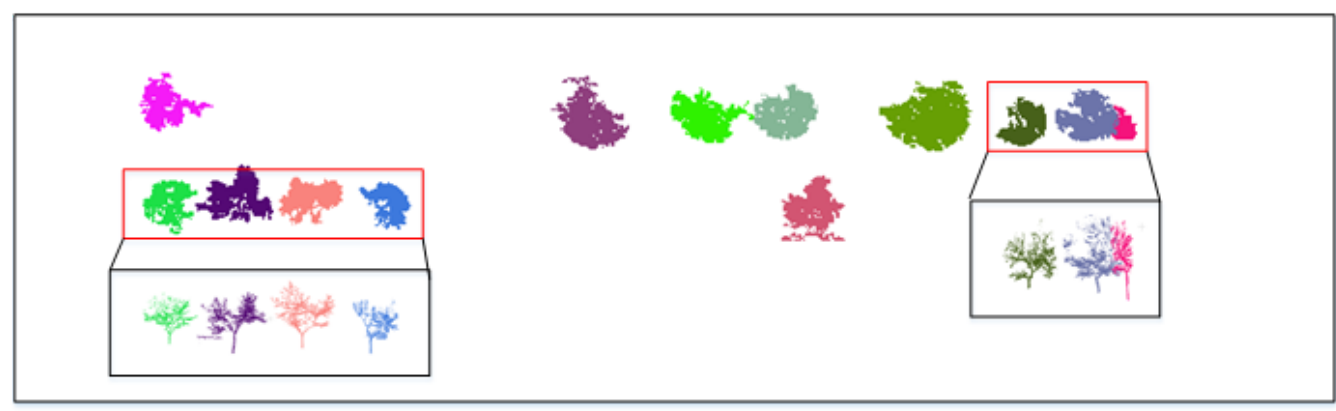

(b)

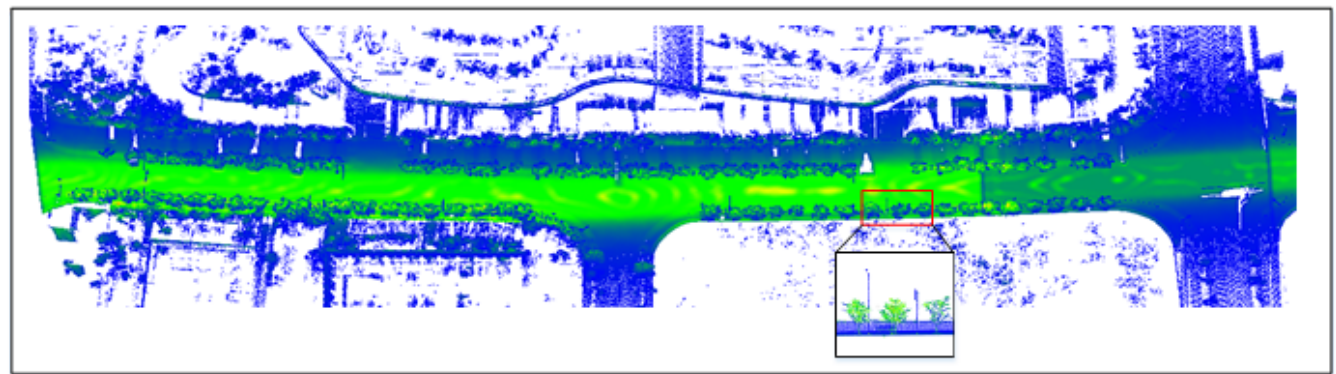

(c)

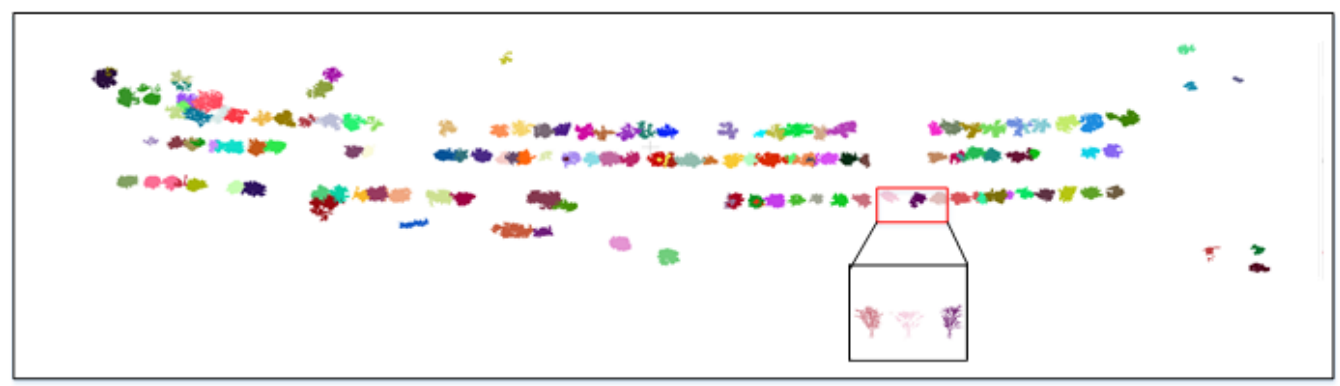

(d)

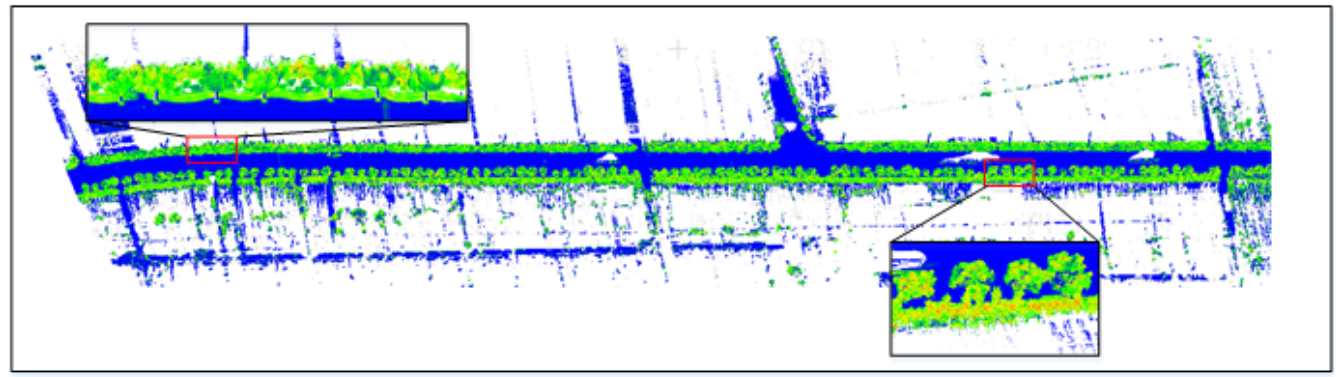

(e)

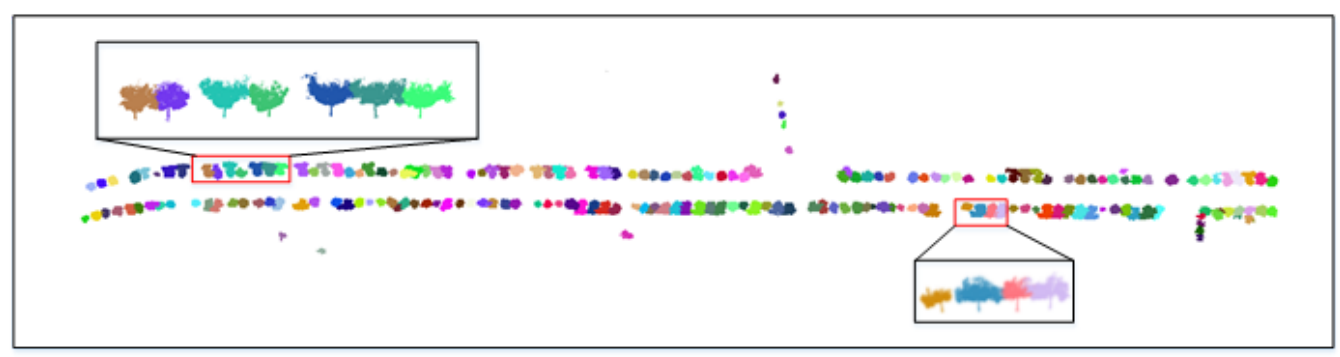

(f)

Figure 2. individual tree extraction: (a) raw data, Huangpu, (b) trees data, Huangpu, (c) raw data, Zhangjiang, (d) trees data, Zhangjiang, (e) raw data, Fengxian, (f) trees data, Fengxian. 